\title{
ABCSG
}




\section{Country: Austria}

Group: $\quad$ Austrian Breast and Colorectal Cancer Study Group (ABCSG)

Chair: $\quad$ M. Gnant

Medical University of Vienna

Department of General Surgery

Währinger Gürtel 18-20

A-1090 VIENNA

AUSTRIA

Tel: +431404005646

Fax: +431404007603

Email: michael.gnant@meduniwien.ac.at

Biostatistics

M. Mittlböck

Unit:

Medical University of Vienna

Core Unit for Medical Statistics and Informatics

Währinger Gürtel 18-20

A-1090 VIENNA

AUSTRIA

Tel: +431404002276

Fax: +431404002278

Email: martina.mittlboeck@meduniwien.ac.at

Study Center: H. Fohler

ABCSG Trial Centre

Boltzmanngasse 24-26, Top 3

A-1090 VIENNA

AUSTRIA

Tel: +4314089230

Fax: +4314090990

Email: hannes.fohler@abcsg.at

Website: $\quad$ www.abcsg.at 
Title:

Adjuvant therapy with CMF versus goserelin plus tamoxifen in premenopausal, hormone-responsive, lymph node-positive or -negative patients.

\section{Study 5}

\section{Coordinator(s): R. Jakesz, M. Gnant}

Medical University of Vienna

Department of General Surgery

Währinger Gürtel 18-20

\section{A-1090 VIENNA}

AUSTRIA

Tel: +431404006916

Fax: +431404006918

Summary: $\quad$ - Opened in December 1990

- Target accrual: 1050 patients

\section{Objectives:}

- To compare DFS and OS in patients treated with standard chemotherapy (cyclophosphamide + methotrexate +5 -fluorouracil) and patients treated with goserelin and tamoxifen.

- To compare toxicities.

Scheme:

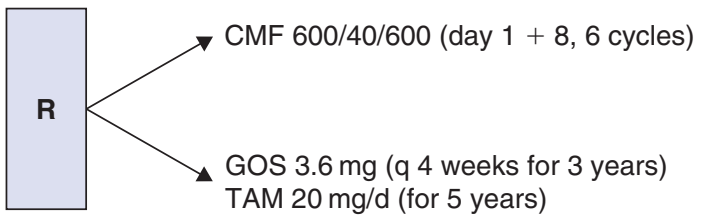

Update: - Study closed in June 1999 with 1034 patients.

- Five-year results were published in 2002.

- Ten-year follow-up analysis in 2006.

Related

Publications:

- Jakesz, et al. Randomized adjuvant trial of tamoxifen and goserelin versus cyclophosphamide, methotrexate, and fluorouracil: evidence for the superiority of treatment with endocrine blockade in premenopausal patients with hormone-responsive breast cancer Austrian Breast and Colorectal Cancer Study Group Trial 5. J Clin Oncol 2002; 20 (24): 4621-4627.
Topics:
- Tamoxifen
- Premenopausal patients
- Hormone receptor-negative breast cancer
- Hormone receptor-positive breast cancer

Keywords: $\quad$ CMF, goserelin, tamoxifen, premenopausal, hormone-responsive, toxicity 
Title:

Adjuvant endocrine therapy in postmenopausal patients with hormoneresponsive breast cancer: tamoxifen versus tamoxifen plus

aminoglutethimide.

\section{Study 6}

Coordinator(s): $\quad$ R. Jakesz

Medical University of Vienna

Department of General Surgery

Währinger Gürtel 18-20

A-1090 VIENNA

AUSTRIA

Tel: +431404006916

Fax: +431404006918

Summary: $\quad$ Opened in December 1990

- Target accrual: 2000

Study 6A:

Re-randomization for recurrence-free patients in Study 6 Anastrozole $1 \mathrm{mg} / \mathrm{d}$ for 3 years versus control

Summary:

- Opened in March 1996

- Target accrual: 812 patients

Objectives:

- To compare addition of aminoglutethimide to endocrine tamoxifen treatment with respect to OS, DFS and side effects (Study 6).

- After 5 years RFS, to compare anastrozole to control with respect to OS and DFS, to assess occurrence of second carcinoma (Study 6A).

Scheme:

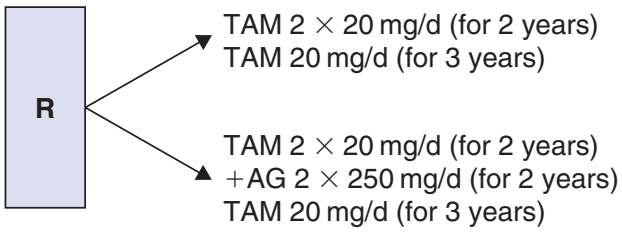

Update: $\quad$ - Study closed in December 1995 with 2021 patients.

- Re-randomization completed in March 2001 with 812 patients.

- Five-year results have been published. 
Related Tamoxifen versus tamoxifen plus aminoglutethimide for stage I and II Publications: receptor-positive postmenopausal node-negative or node-positive breast cancer patients: four-year results of a randomized trial of the Austrian Breast Cancer Study Group (ABCSG). Samonigg H, Jakesz R, Hausmaninger H, et al. P Am Soc Clin Oncol 1999; 18: 68a (Abstract 253).

Substantial increase in the breast conservation rate between 1984 and 1997 in 3464 patients randomized in trials of the Austrian Breast Cancer Study Group (ABCSG). Gnant M, Jakesz R, Hausmaninger H. P Am Soc Clin Oncol 1999; 18: 94a (Abstract 355).

Threefold increase in breast conservation (BC) in 4172 patients accrued during 15 years of trials conducted by the ABCSG. Jakesz R. P Am Soc Clin Oncol 2000; 19: 139a (Abstract 547).

Significant increase in breast conservation in 16 years of trials conducted by the Austrian Breast \& Colorectal Cancer Study Group (ABCSG). Jakesz R, Samonigg H, Gnant M, et al. Ann Surg 2003; 237 (4): 556-564.

Randomized trial of tamoxifen versus tamoxifen plus aminoglutethimide as adjuvant treatment in postmenopausal breast cancer patients with hormone receptor-positive disease: Austrian Breast and Colorectal Cancer Study Group Trial 6. Schmid M, Jakesz R, Samonigg H, et al. J Clin Oncol 2003; 21 (6): 984-990.

Prognostic value of lymphangiogenesis and lymphovascular invasion in invasive breast cancer. Schoppmann SF, Bayer G, Aumayr K, et al. Ann Surg 2004; 240 (2): 306-312.

Topics: - Tamoxifen

- Postmenopausal patients

- Hormone receptor-positive breast cancer

Keywords: Tamoxifen, adjuvant, endocrine, postmenopausal, hormone-responsive, aminoglutethimide 
Title:

Pre and postoperative chemotherapy versus conventional adjuvant chemotherapy alone in patients presenting with hormone receptornegative breast cancer.

\section{Study 7}

Coordinator(s): R. Jakesz

Medical University of Vienna

Department of General Surgery

Währinger Gürtel 18-20

A-1090 VIENNA

AUSTRIA

Tel: +431404006916

Fax: +431404006918

Summary: - Opened in October 1991

- Target accrual: 480 patients

Objectives:

- To compare OS and RFS in patients treated with pre and postoperative chemotherapy versus postoperative chemotherapy alone (cyclophosphamide + methotrexate +5 -fluorouracil and epirubicin + cyclophosphamide).

- To investigate to what percentage breast-conserving surgery may be enhanced by preoperative cytostatis.

Scheme:
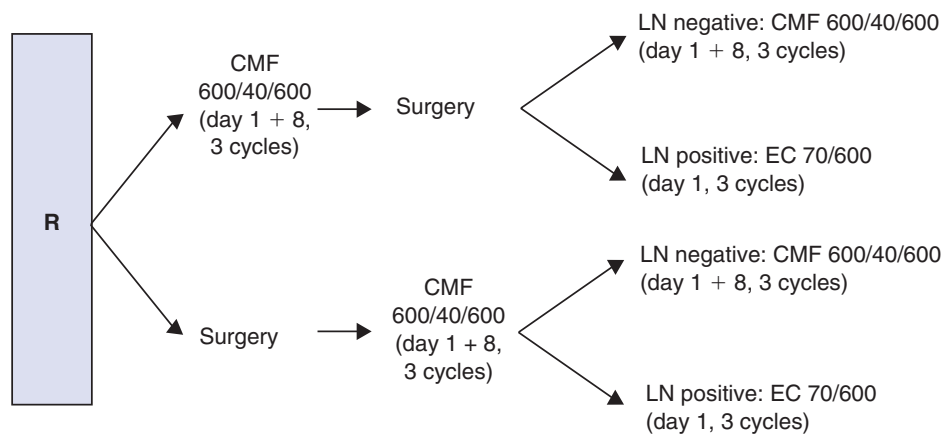

Update: - Study closed in October 1999 with 423 patients.

- Preliminary results were published, in 2001.

Related Comparison of pre versus postoperative chemotherapy in breast cancer Publications: patients: four-year results of Austrian Breast \& Colorectal Cancer Study Group (ABCSG) Trial 7. Jakesz R, for the ABCSG. P Am Society of Clin Oncol 2001; 20: 32a (Abstract 125). 
Sequential steroid hormone receptor measurements in primary breast cancer with and without intervening primary chemotherapy. Taucher $\mathrm{S}$, Rudas M, Gnant M, et al. Endocr-Relat Cancer 2003; 10 (1): 91-98.

Topics: - Node-positive breast cancer

- Node-negative breast cancer

Keywords: Chemotherapy, adjuvant, chemotherapy, hormone receptor-negative, cyclophosphamide, methotrexate, 5-fluorouracil, epirubicin, cyclophosphamide, breast-conserving 
Title:

Adjuvant endocrine therapy in postmenopausal patients with hormoneresponsive breast cancer, G1 and G2 (ARNO).

\section{Study 8}

Coordinator(s): R. Jakesz

Medical University of Vienna

Department of General Surgery

Währinger Gürtel 18-20

A-1090 VIENNA

AUSTRIA

Tel: +431404006916

Fax: +431404006918

Summary: $\quad$ Opened in January 1996

- Target accrual: 3500 patients

Objectives:

- To compare OS, RFS and side effects in postmenopausal patients with primary breast cancer, negative or positive nodes, and well or moderately differentiated tumours, treated with tamoxifen and then randomized to receive either tamoxifen or anastrozole.

- To additionally assess the value of adjuvant radiotherapy following breast-conserving surgery in lymph node-negative patients, $\mathrm{T}<3 \mathrm{~cm}$.

Scheme:

TAM $20 \mathrm{mg} / \mathrm{d}$ (for 2 years)

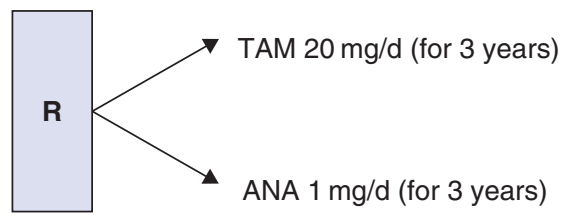

Update: - Study closed in June 2004 with 3500 patients.

- Preliminary results were published, in 2005.

Related

Threefold increase in breast conservation (BC) in 4172 patients

Publications: $\quad$ accrued during 15 years of trials conducted by the ABCSG. Jakesz R. P Am Soc Clin Oncol 2000; 19: 139a (Abstract 547).

Significant increase in breast conservation in 16 years of trials conducted by the Austrian Breast \& Colorectal Cancer Study Group (ABCSG).

Jakesz R, Samonigg H, Gnant M, et al. Ann Surg 2003; 237 (4): 556-564.

Prognostic value of lymphangiogenesis and lymphovascular invasion in invasive breast cancer. Schoppmann SF, Bayer G, Aumayr K, et al. Ann Surg 2004; 240 (2): 306-312. 
Switching of postmenopausal women with endocrine-responsive early breast cancer to anastrozole after 2 years' adjuvant tamoxifen: combined results of ABCSG trial 8 and ARNO 95 trial. Jakesz R, Kaufmann M, Gnant M, et al. The Lancet 2005; 366 (9484): 455-462.

Topics:

- Postmenopausal patients

- Hormone receptor-positive breast cancer

- Breast conservative treatment

- Node-negative breast cancer, radiotherapy

Keywords: Anastrozole, tamoxifen, adjuvant, endocrine, postmenopausal, hormone-responsive, G1, G2, radiotherapy, breast-conserving, lymph node-negative 
Title:

Adjuvant chemotherapy in postmenopausal patients with hormoneresponsive breast cancer, G3.

\section{Study 9}

Coordinator(s): R. Jakesz

Medical University of Vienna

Department of General Surgery

Währinger Gürtel 18-20

A-1090 VIENNA

AUSTRIA

Tel: +431404006916

Fax: +431404006918

Summary: $\quad$ - Opened in January 1996

- Target accrual: 660 patients

Objective:

To compare OS, RFS and side effects in postmenopausal patients with primary breast cancer, negative or positive nodes, and undifferentiated, tumours, treated with adjuvant standard tamoxifen or additional epirubicin + cyclophosphamide.

Scheme:

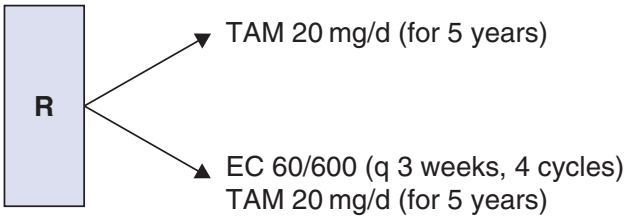

Update:

- Study closed in December 2005 with 660 patients.

Related

Threefold increase in breast conservation (BC) in 4172 patients

Publications: accrued during 15 years of trials conducted by the ABCSG. Jakesz R. P Am Soc Clin Oncol 2000; 19: 139a (Abstract 547).

Significant increase in breast conservation in 16 years of trials conducted by the Austrian Breast \& Colorectal Cancer Study Group (ABCSG). Jakesz R, Samonigg H, Gnant M, et al. Ann Surg 2003; 237 (4): 556-564.

Impact of pretreatment thrombocytosis on survival in primary breast cancer. Taucher S, Salat A, Gnant M, et al. Thromb Haemostasis 2003; 89 (6): 1098-1106.

Prognostic value of lymphangiogenesis and lymphovascular invasion in invasive breast cancer. Schoppmann SF, Bayer G, Aumayr K, et al. Ann Surg 2004; 240 (2): 306-312. 
Topics:

- Hormone receptor-positive breast cancer

- Postmenopausal patients

- Tamoxifen

Keywords: Adjuvant, chemotherapy, postmenopausal, hormone-responsive, tamoxifen, epirubicin, cyclophosphamide, G3 
Title:

Adjuvant endocrine therapy and bisphosphonate therapy: tamoxifen in comparison to anastrozole, alone or in combination with zoledronate, in premenopausal patients presenting with hormone-responsive, Stage I and II breast cancer.

\section{Study 12}

Coordinator(s): R. Jakesz

Medical University of Vienna

Department of General Surgery

Währinger Gürtel 18-20

A-1090 VIENNA

AUSTRIA

Tel: +431404006916

Fax: +431404006918

Summary: $\quad$ - Opened in June 1999

- Target accrual: 1250 patients

Objectives:

- To compare RFS and OS in patients treated with tamoxifen or anastrozole.

- To assess whether bisphosphonate treatment with zoledronate added to standard adjuvant therapy may improve RFS and OS.

Scheme:

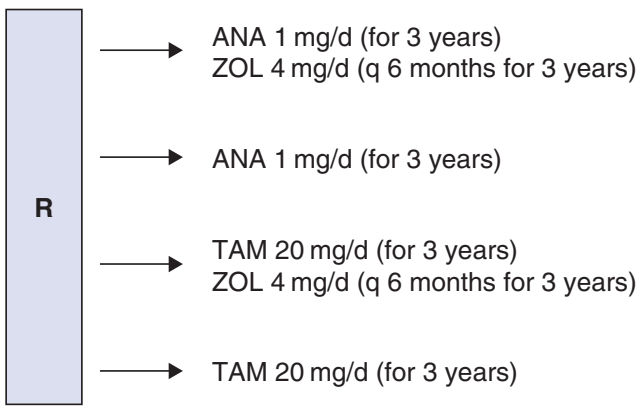

All patients receive basic therapy with goserelin $3.6 \mathrm{mg}$ ( $q 4$ weeks for 3 years)

Update: - Increase of target accrual to bone mineral density sub-protocol from 200 to 400 patients.

- Increase of target accrual to main protocol from 1250 to 1800 patients.

- Study closed in May 2006 with 1800 patients. 
Related

Publications:

Topics:

Keywords:
Amenorrhea, aromatase inhibitors, tamoxifen or a combination in premenopausal women. Jakesz R. Eur J Cancer 2004; 2 (9): 51-53.

Zoledronic acid effectively prevents cancer treatment-induced bone loss in premenopausal women receiving adjuvant endocrine therapy for hormone-responsive breast cancer. Gnant M, Mlineritsch B, LuschinEbengreuth G, et al. J Clin Oncol [In press].

- Bisphosphonates

- Bone mineral density

- Hormonal therapy

- Hormone receptor-positive breast cancer

- Premenopausal patients

- Tamoxifen

Adjuvant, endocrine, bisphosphonate, tamoxifen, anastrozole, zoledronate, premenopausal, hormone-responsive, Stage I, Stage II 
Title:

Effect of 3 versus 6 cycles of epidoxorubicin/docetaxel + G-CSF upon the rate of complete pathological remissions in the neoadjuvant treatment of patients with primary breast cancer and no distant metastases.

\section{Study 14}

\section{Coordinator(s): G. Steger}

Medical University of Vienna

Department of Oncology

Währinger Gürtel 18-20

A-1090 VIENNA

AUSTRIA

Tel: +431404004426

Fax: +431404004451

Summary: $\quad$ Opened in February 2001

- Target accrual: 282 patients

Objectives:

- To assess the rate of pathological complete remissions.

- To assess the rates of axillary lymph node metastases and breastconserving procedures following 3 versus 6 cycles of epidoxorubicin (short infusion)/docetaxel (1-hour infusion).

Scheme:

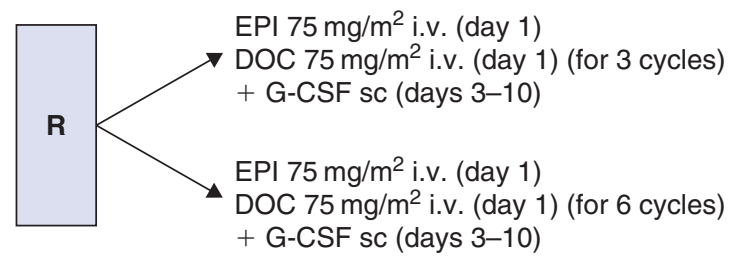

Update: - Study closed in December 2002 with 292 patients.

Related None available

Publications:

Topics: - G-CSF secondary prophylaxis, breast conservative treatment

Keywords: $\quad$ Epidoxorubicin, docetaxel, G-CSF, complete pathological remission, neoadjuvant, breast-conserving, metastases, lymph node 
Title:

SALSA (Secondary Adjuvant Long-term Study with Arimidex): a prospective, randomized, open, multicentre Phase III study to assess the efficacy of secondary adjuvant endocrine anastrozole therapy for 2 further years versus 5 further years in patients with hormone receptorpositive breast cancer after a 5-year primary adjuvant endocrine therapy.

\section{Study 16}

\section{Coordinator(s): M. Gnant}

Medical University of Vienna

Department of General Surgery

Währinger Gürtel 18-20

A-1090 VIENNA

AUSTRIA

Tel: +431404005646

Fax: +431404007603

Email: michael.gnant@meduniwien.ac.at

Summary: $\quad$ Opened in February 2004

- Target accrual: 3500 patients

Primary Objective:

- Assessment of the effect of 2 further years of anastrozole treatment versus further 5 years of anastrozole treatment on DFS, following a 5-year adjuvant endocrine therapy.

\section{Secondary Objectives:}

- Assessment of the effect of further 2 years of anastrozole versus further 5 years anastrozole treatment on the overall survival rate following a 5-year adjuvant endocrine therapy.

- Comparison of the respective rates of fracture occurrence in the two groups.

- Comparison of the occurrence (a) of secondary carcinoma, except in the case of a contralateral mammary carcinoma (b) of a contralateral breast cancer in the two groups, respectively.

Scheme:

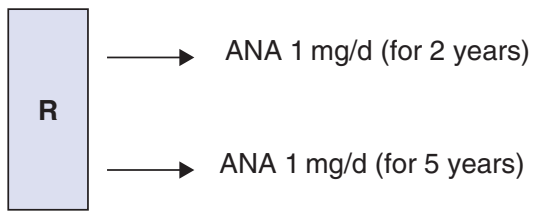

All patients received a 5-year primary adjuvant endocrine therapy 
Update:

Related

Publications:

Topics:

Keywords:
- 1327 patients entered until February 2006.

None available

- Aromatase inhibitors

- Hormonal therapy

- Hormone receptor-positive breast cancer

Arimidex, anastrozole, adjuvant, long-term, receptor-positive 
Title: Neoadjuvant hormonal therapy with exemestane in postmenopausal patients with primary hormone receptor-positive breast cancer and no distant metastases.

\section{Study 17}

Coordinator(s): R. Greil

III. Medical Department

General Hospital/Paracelsus University Salzburg

Müllner Hauptstraße 48

A-5020 SALZBURG

AUSTRIA

Tel: +43662 44822879

Fax: +43662 44822898

Summary: $\quad$ Opened in September 2000

- Target accrual: 95 patients

Objective:

- To evaluate efficacy in terms of clinical response (complete response, partial response, no change).

Scheme:

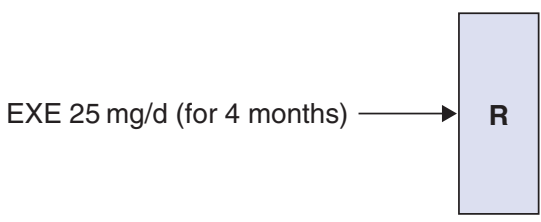

Exemestane $25 \mathrm{mg} / \mathrm{d}$ (for 4 months)

Update: - Study closed in October 2003 with 95 patients.

Related None available

Publications:

Topics: $\quad$ Hormone receptor-positive breast cancer

- Postmenopausal patients

Keywords: Neoadjuvant, exemestane, postmenopausal, receptor-positive, response 
Title:

A randomized, double-blind, placebo-controlled, multi-centre phase 3 study to determine the treatment effect of denosumab in subjects with non-metastatic breast cancer receiving aromatase inhibitor therapy.

Study 18

Coordinator(s): M. Gnant

Medical University of Vienna

Department of General Surgery

Währinger Gürtel 18-20

A-1090 VIENNA

AUSTRIA

Tel: +431404005646

Fax: +431404007603

Email: michael.gnant@meduniwien.ac.at

Summary: $\quad$ - Ready to be opened (scheduled for October 2006)

- Target accrual: 2800 patients

Primary Objective:

- To determine whether denosumab compared to placebo will reduce the rate of first clinical fracture in women with non-metastatic breast cancer receiving (non-steroidal) aromatase inhibitor therapy (AIT).

Secondary Objectives:

- To assess the effect of denosumab compared to placebo on the following: Incidence of new vertebral fractures (both clinical and morphometric). Incidence of new or worsening of pre-existing vertebral fractures (both clinical and morphometric)

- Bone mineral density (BMD) at lumbar spine, total hip and femoral neck in a subgroup of subjects at pre-selected sites

- To assess the safety and tolerability of denosumab in this population 
Scheme:
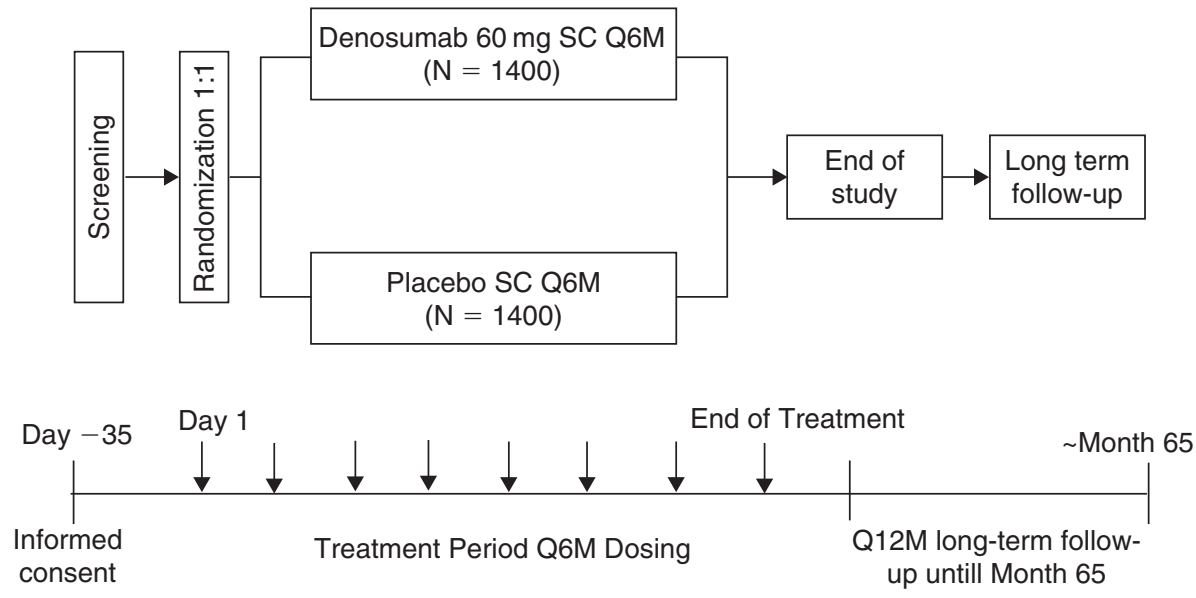

Update: $\quad$ None available

Related None available

Publications:

Topics: $\quad$ Aromatase inhibitors

- Bone mineral density

Keywords: Denosumab, placebo, aromatase inhibitor therapy, bone mineral density, vertebral fractures, non-metastatic breast cancer 
Title:

A randomised Phase II study comparing anastrozole and fulvestrant to anastrozole for adjuvant treatment of postmenopausal patients with early breast cancer and disseminated tumour cells in bone marrow.

\section{Study 21}

Coordinator(s): S. Braun

University Hospital Innsbruck

Department for Obstetrics and Gynecology

Anichstraße 35

A-6020 INNSBRUCK

AUSTRIA

Tel: +4351250481194

Fax: +436641215765

Email: stephan.braun@uibk.ac.at

Summary: $\quad$ Opened in April 2006

- Target accrual: 176 patients

\section{Objectives:}

- To compare the frequency of events (presence of DTC, clinical recurrence and/or death) after 12 months of randomised treatment (primary).

- To assess the safety of the anastrozole-fulvestrant combination (secondary).

- To assess the frequency of events (presence of DTC, clinical recurrence and/or death) after 24 months of randomised treatment (secondary).

- To explore the clinical utility of comparing the numbers of DTC in patients before and after 12 and 24 months of randomised treatment by evaluating the degree of any DTC reduction in relation to clinical recurrence and/or death (exploratory).

- To explore the clinical utility (occurrence versus absence of clinical event) of a molecular approach for the assessment of disseminated ribonucleotides in BM as compared to immunocytochemistry in BM before and after 12 and 24 months of randomised treatment (exploratory).

- To explore the clinical utility (occurrence versus absence of clinical event) of a molecular approach for the assessment of circulating ribonucleotides in blood as compared to immunocytochemistry in BM before and after 12 and 24 months of randomised treatment (exploratory).

- To explore the clinical utility (occurrence versus absence of clinical event) of a molecular approach for the assessment of circulating methylated DNA in blood and BM as compared to immunocytochemistry in BM before and after 12 and 24 months of randomised treatment (exploratory). 
- To explore the expression of hormone receptors, HER-2 oncoprotein and broad stem cell characteristics of DTC before and after 12 and 24 months of randomised treatment (exploratory).

Scheme:

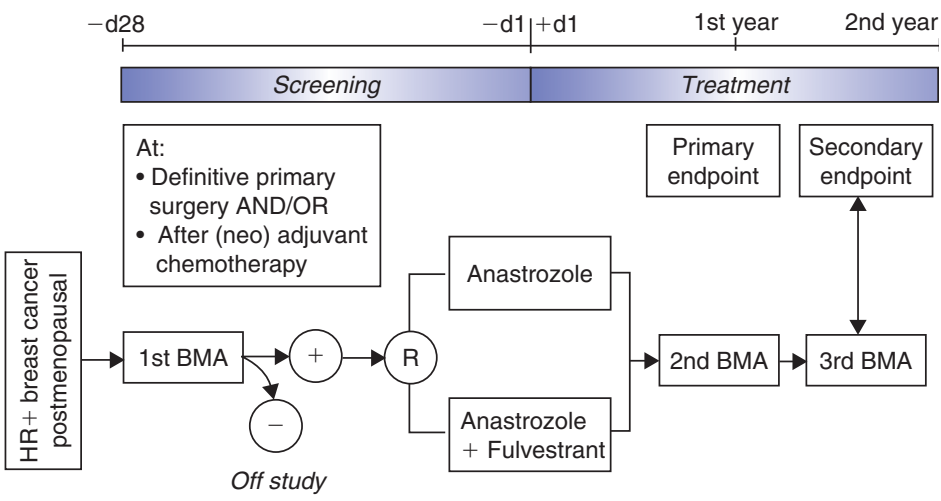

Update: $\quad$ None available

Related None available

Publications:

Topics:

- Blood markers

- Aromatase inhibitors

- Bone marrow micrometastasis

- Postmenopausal patients

Keywords: Phase II, anastrozole, fulvestrant, adjuvant treatment, postmenopausal, early breast cancer, disseminated tumour cells, bone marrow, ribonucleotides, HER-2, immunocytochemistry 
Title:

A randomized Phase III study comparing epirubicin, docetaxel and capecitabine + G-CSF to epirubicin and docetaxel + G-CSF as neoadjuvant treatment for early HER-2 negative breast cancer and comparing epirubicin, docetaxel and capecitabine + G-CSF \pm trastuzumab to epirubicin and docetaxel + G-CSF \pm trastuzumab as neoadjuvant treatment for early HER-2 positive breast cancer.

\section{Study 24}

\section{Coordinator(s): G. Steger}

Medical University of Vienna

Department of Oncology

Währinger Gürtel 18-20

A-1090 VIENNA

AUSTRIA

Tel: +431404004426

Fax: +431404004451

Email: guenther.steger@meduniwien.ac.at

Summary: $\quad$ - Opened in November 2004

- Target accrual: 460 patients

\section{Objectives:}

- The primary aim is to evaluate the rate (percentage) of pathological complete remissions at the time of final surgery after 6 cycles in Arm A (Epirubicin/Docetaxel/Capecitabine-containing chemotherapy \pm trastuzumab in HER-2 positive disease) versus Arm B (Epirubicin/Docetaxel/-containing chemotherapy \pm trastuzumab in HER-2 positive disease).

- A secondary aim is to evaluate the rate (percentage) of axillary lymph node involvement at the time of final surgery in Arm A ( \pm trastuzumab in HER-2 positive disease) versus Arm B ( \pm trastuzumab in HER-2 positive disease).

- Another secondary aim is to evaluate the rate (percentage) of breastconserving procedures at the time of final surgery in Arm A ( \pm trastuzumab in HER-2 positive disease) versus Arm B ( \pm trastuzumab in HER-2 positive disease). 
Scheme:

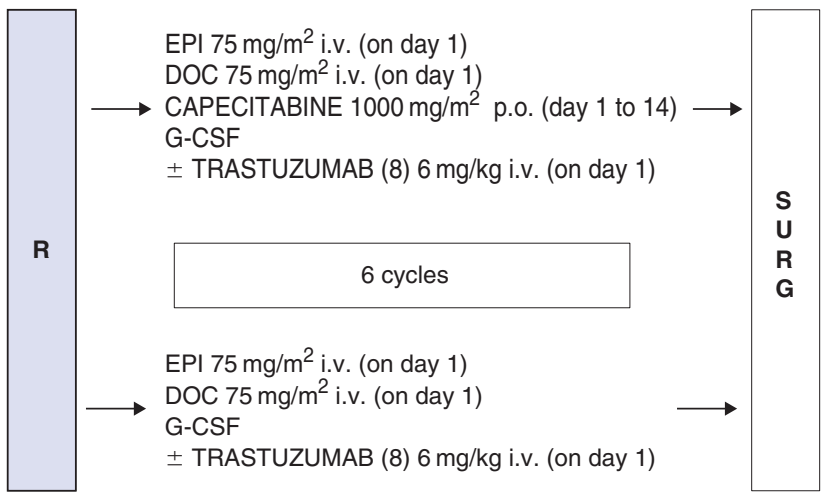

Patients with HER-2 positive tumours without a neoadjuvant trastuzumab therapy participate in an adjuvant trastuzumab therapy for 12 months (18 applications). Patients with HER-2 positive tumours receiving a neoadjuvant trastuzumab therapy participate in an adjuvant trastuzumab therapy 8 months (12 applications).

Update:

- June 2005 Addendum: determination of E-cadherin serum levels in breast cancer patients with neoadjuvant therapy entered into the ABCSG - Protocol 24l.

- November 2005: Addendum: assessment of disseminated and circulating tumour cells in breast cancer patients with neoadjuvant therapy assigned to the ABCSG - Protocol 24.

- March 2006 Amendment: protocol \pm neoadjuvant therapy with trastuzumab for HER-2 positive patients.

- March 2006 Addendum: assessment of disseminated and circulating tumour cells in breast cancer patients with neoadjuvant therapy assigned to the ABCSG - Protocol 24.

- 140 patients entered until February 2006.

Related None available

Publications:

Topics:

- Capecitabine

- Breast conservative treatment

- G-CSF secondary prophylaxis

- Blood markers

- Bone marrow micrometastasis

- HER-2 negative patients

- HER-2 positive patients

- Trastuzumab

Keywords: $\quad$ Epirubicin, docetaxel, capecitabine, G-CSF, trastuzumab, neoadjuvant, HER-2, pathological complete remissions, axillary lymph node involvement, chemotherapy 